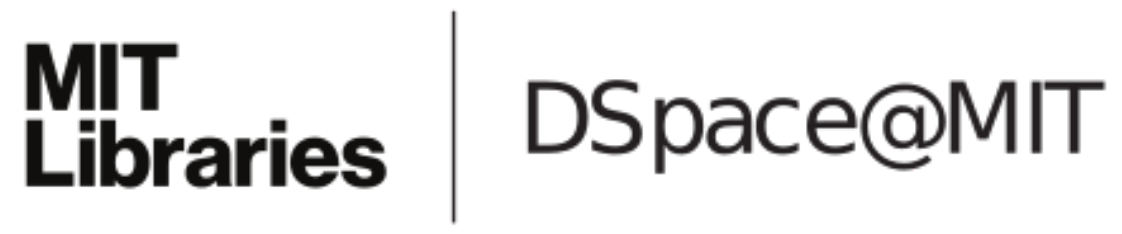

MIT Open Access Articles

Tuning Material Properties of Nanoemulsion Gels by Sequentially Screening Electrostatic Repulsions and Then Thermally Inducing Droplet Bridging

The MIT Faculty has made this article openly available. Please share how this access benefits you. Your story matters.

Citation: Cheng, Li-Chiun, Signe Lin Kuei Vehusheia and Patrick S. Doyle. “Tuning Material Properties of Nanoemulsion Gels by Sequentially Screening Electrostatic Repulsions and Then Thermally Inducing Droplet Bridging" Langmuir, vol. 36, no. 13, 2020, pp. 3346-3355 ๑ 2020 The Author(s)

As Published: https://dx.doi.org/10.1021/acs.langmuir.0c00199

Publisher: American Chemical Society (ACS)

Persistent URL: https://hdl.handle.net/1721.1/125656

Version: Final published version: final published article, as it appeared in a journal, conference proceedings, or other formally published context

Terms of use: Creative Commons Attribution 4.0 International license 


\title{
Tuning Material Properties of Nanoemulsion Gels by Sequentially Screening Electrostatic Repulsions and Then Thermally Inducing Droplet Bridging
}

\author{
Li-Chiun Cheng, Signe Lin Kuei Vehusheia, and Patrick S. Doyle*
}

Cite This: Langmuir 2020, 36, 3346-3355

Read Online

ACCESS | Llll Metrics \& More | 期 Article Recommendations

S1 Supporting Information

ABSTRACT: Nanoemulsions are widely used in applications such as food products, cosmetics, pharmaceuticals, and enhanced oil recovery for which the ability to engineer material properties is desirable. Moreover, nanoemulsions are emergent model colloidal systems because of the ease in synthesizing monodisperse samples, flexibility in formulations, and tunable material properties. In this work, we study a nanoemulsion system previously

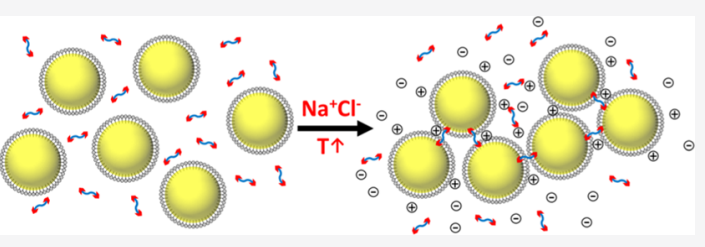
developed by our group in which gelation occurs through thermally induced polymer bridging of droplets. We show here that the same system can undergo a sol-gel transition at room temperature through the addition of salt, which screens the electrostatic interaction and allows the system to assemble via depletion attraction. We systematically study how the addition of salt followed by a temperature jump can influence the resulting microstructures and rheological properties of the nanoemulsion system. We show that the salt-induced gel at room temperature can dramatically restructure when the temperature is suddenly increased and achieves a different gelled state. Our results offer a route to control the material properties of an attractive colloidal system by carefully tuning the interparticle potentials and sequentially triggering the colloidal self-assembly. The control and understanding of the material properties can be used for designing hierarchically structured hydrogels and complex colloid-based materials for advanced applications.

\section{INTRODUCTION}

Colloidal gelation is an effective tool to engineer material properties. By properly inducing the gelation of colloidal suspensions, one can precisely modulate the macroscopic properties and create complex microstructures. ${ }^{1}$ For example, colloidal suspensions that undergo gelation can be used as rheology modifiers ${ }^{2}$ or ingredients in pharmaceutical $^{3-5}$ and food products, ${ }^{6,7}$ and the complex structures can be used as scaffolds in tissue engineering ${ }^{8,9}$ or porous material design. ${ }^{10}$ Among a diverse set of colloidal suspensions, nanoemulsions have been an emerging model colloidal system for studying aspects of colloidal gel systems. ${ }^{11}$ Nanoemulsions are liquidliquid suspensions (e.g., oil droplets suspended in water) where the droplet size is on the order of $100 \mathrm{~nm}$. The ease of nanoemulsion synthesis and the great flexibility in their formulation allow one to engineer the inter-droplet potentials and thus to rationally tune material microstructures and associated macroscopic properties. ${ }^{12}$ Therefore, gelling nanoemulsions have attracted much attention in both fundamental studies $^{13-15}$ and practical applications. ${ }^{16-18}$

Several strategies have been applied to induce the gelation of nanoemulsions. One common approach to trigger gelation is by depletion interaction. By adding nonadsorbing molecules into the continuous phase of the nanoemulsions, the molecules are excluded from the vicinity of the droplets because of increase of entropy. ${ }^{19-21}$ Such exclusion gives rise to an imbalance in the osmotic pressure, leading to a net attractive interaction between the droplets and ultimately gelation of the system. Different types of nonadsorbing depletant molecules such as polymers ${ }^{15,16}$ and surfactant micelles ${ }^{22,23}$ have been employed to induce self-assembly and gelation of nanoemulsions. Another well-known approach to obtain gelling nanoemulsions is to modulate the effective range of the interdroplet electrostatic repulsion. ${ }^{14,24,25}$ By adding electrolytes to the charged-stabilized nanoemulsions (e.g., stabilized by ionic surfactants), the ions from the dissociating electrolytes screen the electrostatic repulsion by decreasing the Debye length. ${ }^{26,27}$ If enough electrolytes are added into the nanoemulsions, a secondary minimum can be obtained in the pairwise interactive potentials and the system can self-assemble and eventually gel. $^{24,25}$ Recently, our group has designed another gelling nanoemulsion mechanism in which the gelation is responsive to an increase in temperature. ${ }^{28,29}$ In this approach, telechelic oligomers are added to the continuous phase and their hydrophobic end groups will preferentially partition into the oil domains upon an increase in temperature, resulting in interdroplet bridging. The polymer bridging acts as an effective

Received: January 23, 2020

Revised: March 17, 2020

Published: March 27, 2020 
attractive force between the droplets and ultimately gives rise to gelation at high enough temperatures.

Although we have done many studies on this canonical bridging system, in the prior work, we only focused on the bridging mechanism ${ }^{29}$ and used the resulting nanoemulsion gels to explore different topics related to colloidal gels. For example, we have used this model system to build the gel phase diagram and explored the gelation routes (percolation vs phase separation), ${ }^{28,30}$ applied multiple particle tracking to probe colloid-rich and colloid-lean phases ${ }^{31}$ and studied the effect of processing history on gel properties. ${ }^{32}$ Other researchers have also used this same canonical system to explore the dynamics during colloidal gelation ${ }^{33}$ and gel behaviors in the nonlinear regime. ${ }^{34}$ Although in these aforementioned studies the bridging nanoemulsion system is used as a model colloidal gel system, the quantitative details of the droplet interactions were not well understood and depletion interactions in the system were largely ignored. In a recent study, ${ }^{15}$ we found that even at room temperature where the bridging is not triggered, the free polymers in the nanoemulsion system provide a substantial depletion interaction (strength $\approx 15 \mathrm{kT}$ ). This depletion attraction is delicately compensated by the electrostatic repulsion given by the ionic surfactant on the droplets. This delicate competition was ignored in all previous studies on this canonical system. Therefore, in this work, one of our primary goals is to have a quantitative understanding of these competing interactions at room temperature and to build the interactive potentials from first principles. This is achieved by the addition of salt to suppress the electrostatic repulsions, and it naturally serves as our first gelation route.

Furthermore, in prior colloidal gel studies, gelation is induced by a single gelation route (i.e., pure depletion, electrolyte screening, or droplet bridging). To obtain more diverse material behaviors, a sequential application of different gelation routes to the nanoemulsion could be beneficial. The system we developed is a practical way to design multistimuli responsive materials. Overall, there is an increasing need to transition from traditional chemistry-based methods to physicochemical methods for material design. Systems which respond to multiple stimuli inherently have a more diverse set of achievable material properties. By realizing the depletion interaction is significant at room temperature, the change in ionic strength serves as the first stimulus to tune the material properties. We then use the increase in temperature as the second stimulus to the system. The sequential application of these two stimuli allows us to have a well-controlled system. Furthermore, it builds upon the well-studied depletion-induced colloidal gelation literature and demonstrates how these systems can be reconfigured with a mere change in temperature.

The nanoemulsion studied here consists of polydimethylsiloxane (PDMS) nanodroplets suspended in an aqueous continuous phase containing poly(ethylene glycol) diacrylate (PEGDA). PEGDA is the thermally-triggered telechelic polymer bridging agent where the PEG segment is the hydrophilic backbone and the acrylate groups at the ends are the hydrophobic moieties. The PDMS nanodroplets are stabilized by an anionic surfactant, sodium dodecyl sulfate (SDS), which provides a charged surface on the droplets that allows us to screen the electrostatic repulsion by adding electrolytes into the system.

To carry out the study, we first added the electrolyte (sodium chloride, $\mathrm{NaCl}$ ) to the nanoemulsion system to induce the first-stage gelation by screening the electrostatic repulsion. We showed that the system can undergo a sol-gel transition at room temperature with a small amount of $\mathrm{NaCl}$, similar to prior published studies. ${ }^{24,35,36}$ We quantitatively investigated the system by calculating the inter-droplet pairwise interactions under the effect of the screened electrostatic repulsion. We discovered that the nonadsorbing PEGDA (as the bridging is not induced at room temperature) gives rise to a significant depletion attraction, and thus a slight increase in the ionic strength can result in gelation of the system. After preconditioning the nanoemulsion with $\mathrm{NaCl}$ at room temperature, we then increased the temperature to induce PEGDA droplet bridging. Our results show a nonintuitive trend in the material properties in which a stronger gel is not necessarily obtained with this "two-stage" gelation. We also showed that the screening of electrostatic repulsions at room temperature in the first step has a considerable influence on the subsequent nanoemulsion microstructures and the associated rheological properties at elevated temperatures. We then used the established pairwise interactive potential and the dynamics of the thermal gelation to explain the trends in material behavior under the sequential application of stimuli. Our results suggest that sequential droplet self-assembly can be an effective tool to engineer properties of colloid-based materials, and an understanding of the molecular behavior of the constituents is critical for designing self-assembling systems. The designed pathway could also aid in practical applications such as personal care products. For example, nanoemulsion gels can be used as creams or lotions applied to skin. ${ }^{16}$ Under such conditions, the nanoemulsion will experience a change in ionic strength and temperature, and it is therefore important to understand the material properties subjected to these multiple stimuli.

\section{MATERIALS AND METHODS}

2.1. Materials. PDMS (viscosity $=5 \mathrm{cSt})$, SDS, PEGDA $\left(M_{\mathrm{n}} \approx\right.$ $\left.700 \mathrm{~g} \mathrm{~mol}^{-1}\right)$, sodium chloride $(\mathrm{NaCl})$, lipophilic dye $\mathrm{PKH} 26$ (excitation and emission wavelengths $\lambda_{\mathrm{ex}} / \lambda_{\mathrm{em}}=551 / 567 \mathrm{~nm}$ ), and photoinitiator 2-hydroxy-2-methylpropiophenone (Darocur 1173) were purchased from Sigma-Aldrich. All chemicals were used without further purification.

2.2. Nanoemulsion Synthesis. The nanoemulsion studied in this work consists of a disperse phase PDMS of a volume fraction $\phi=0.15$ $($ droplet diameter $=36 \mathrm{~nm}$ with polydispersity $=0.18)$ suspended in a continuous phase composed of PEGDA of a volume fraction $=0.33$ and SDS. The total concentration of SDS is $0.175 \mathrm{M}$. The concentration of $\mathrm{NaCl}$ is adjusted by adding $\mathrm{NaCl}$ into the nanoemulsion after the synthesis.

The nanoemulsion was synthesized using a high-pressure homogenizer (EmulsiFlex-C3, Avestin). ${ }^{18,28-30}$ Before the homogenization, a pre-emulsion was first prepared by adding PDMS into a premixed continuous phase consisting of deionized water, SDS, and PEGDA. The mixture was stirred using magnetic stirring with a speed of $700 \mathrm{rpm}$. Stirring was maintained for $15 \mathrm{~min}$ and no macroscopic phase separation was observed. The pre-emulsion was subsequently processed into nanoemulsions using high-pressure homogenization at a pressure of $18 \mathrm{kpsi}$ for 16 passes. The emulsion was cooled to $4{ }^{\circ} \mathrm{C}$ between each pass and the final nanoemulsion (pure nanoemulsion without addition of $\mathrm{NaCl}$ ) was stored at $4{ }^{\circ} \mathrm{C}$ until further use. $\mathrm{NaCl}$ with different target concentrations was then added to the pure nanoemulsion and mixed by vortexing immediately before the characterization. The small amount of $\mathrm{NaCl}$ added has a negligible effect on the volume fraction of the nanoemulsions.

The size of nanoemulsions was measured using dynamic light scattering (90Plus PALS, Brookhaven Instruments). Before the measurement, the nanoemulsion was diluted from $\phi=0.15$ to $\phi=$ 


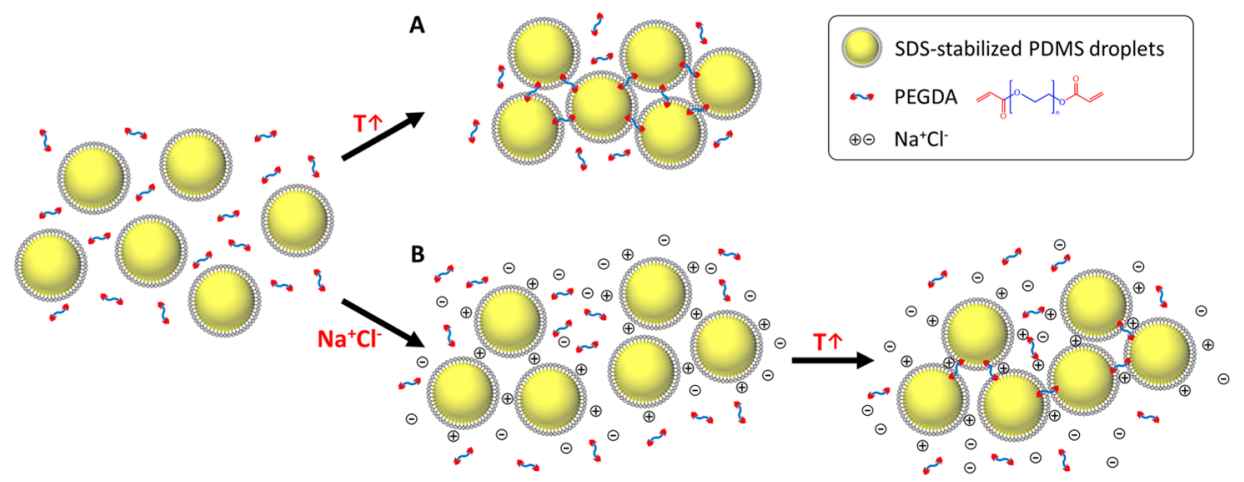

Figure 1. Schematic of the gelation routes studied in this work. (A) Upper route: thermal gelation via PEGDA bridging at elevated temperatures. (B) Lower route: sequentially screening the electrostatic repulsion by adding $\mathrm{NaCl}$ and then inducing thermal bridging with PEGDA droplet bridging.
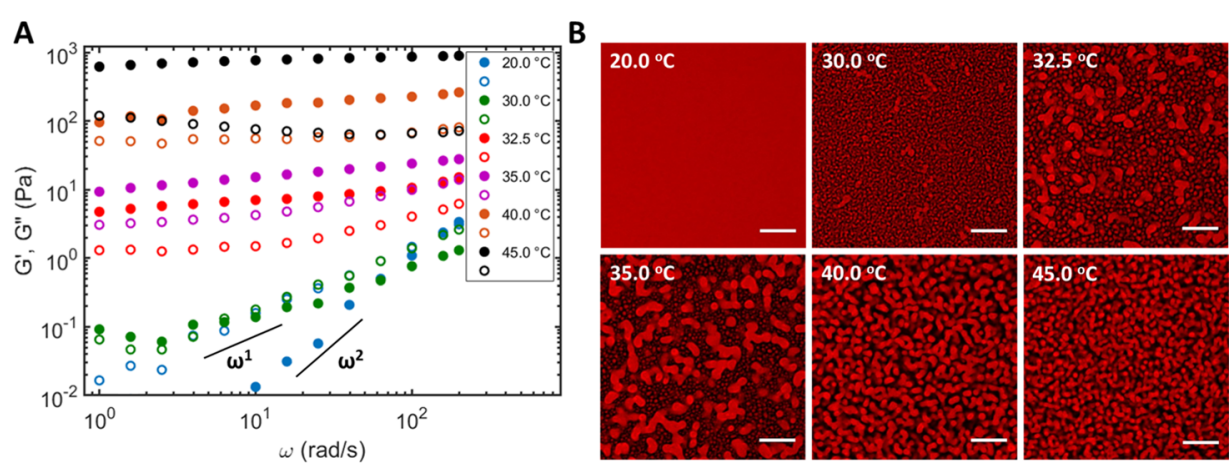

Figure 2. Rheological response and the associated microstructures of the nanoemulsion at elevated temperatures. $[\mathrm{NaCl}]=0 \mathrm{M}$. (A) Linear viscoelastic moduli (closed symbols are storage moduli $G^{\prime}$, open symbols are loss moduli $G^{\prime \prime}$ ) as a function of angular frequency $\omega$. (B) Microstructure of the assembled nanoemulsions at elevated temperatures. The droplets are fluorescently labeled using a lipophilic dye and imaged using confocal microscopy. Scale bars $=10 \mu \mathrm{m}$.

0.002 using an aqueous diluting agent composed of PEGDA at a volume fraction $=0.33$.

2.3. Rheological Measurements. All rheological measurements were conducted using a stress-controlled rheometer (DHR-3, TA instrument) equipped with a $1^{\circ} 60 \mathrm{~mm}$ aluminum upper-cone and a Peltier lower-plate to control the sample temperature. For each measurement, the nanoemulsion was loaded onto the lower plate at $20{ }^{\circ} \mathrm{C}$. To minimize evaporation, a wetted solvent trap was used and a few drops of deionized water were added on the top of the uppercone. Before each measurement, a rejuvenation step was performed by applying constant shear at a shear rate of $2500 \mathrm{~s}^{-1}$ for $60 \mathrm{~s}$ followed by a $10 \mathrm{~min}$ period where the sample remained quiescent at $T=20.0^{\circ} \mathrm{C}$. By studying the flow curves of the nanoemulsions, we showed that at such a high shear rate the gel structure appears to be broken down, especially for nanoemulsions with added salt for which gelation is induced by screening the electrostatic repulsion (Figure S1). The gel structure can then be recovered after $10 \mathrm{~min}$ by monitoring the viscoelastic moduli (Figure S3). Our rejuvenation protocol using high shear rates is consistent with prior work by Wilking et al. ${ }^{25}$ who studied the rheology-structure relation of nanoemulsion gels.

Frequency sweep measurements were then performed after the rejuvenation step. Small-amplitude oscillatory shear (SAOS) was applied to measure the dynamic viscoelastic modulus at a shear strain $=0.05 \%$ with angular frequency $\omega=1-200 \mathrm{rad} \mathrm{s}^{-1}$. For the measurements at different temperatures, the sample was raised from $T$ $=20.0^{\circ} \mathrm{C}$ to the target temperature and then remained quiescent for $10 \mathrm{~min}$ before applying SAOS. Freshly loaded nanoemulsion was used for each measurement.

2.4. Confocal Microscopy. Microstructures of the soft materials dictate rheological properties. ${ }^{37,38}$ In this work, direct visualization of the nanoemulsion microstructures under different conditions was performed using a confocal microscope (LSM 700, Zeiss) equipped with a $63 \mathrm{X}$ oil-immersion objective (numerical aperture $=1.4$ ). To prepare the sample for imaging, $1 \mathrm{vol} \%$ of fluorescent dye and $1 \mathrm{vol} \%$ of photoinitiator were mixed with the nanoemulsion sample by vortexing for $60 \mathrm{~s}$. It has been shown in our previous work that the addition of these chemicals has a negligible effect on the microstructures. ${ }^{31,32}$ Subsequently, $150 \mu \mathrm{L}$ of the mixture was loaded into a glass chamber (Lab-Tek \#155411, Thermo Fisher Scientific) followed by a 10 min period where the sample remained quiescent at $T=20.0$ ${ }^{\circ} \mathrm{C}$. For the samples at elevated temperatures, the mixture was placed in the oven at the target $T$ for $10 \mathrm{~min}$. The sample was subsequently exposed to UV light $(\lambda=365 \mathrm{~nm})$ for $50 \mathrm{~s}$ in the oven. The crosslinking of the excess PEGDA in the continuous phase then locked the assembled microstructure in place, allowing for confocal imaging at room temperature. ${ }^{30-32}$

All characterizations (rheology and confocal microscopy) in this work were performed on the same stock nanoemulsion.

\section{RESULTS AND DISCUSSIONS}

Figure 1 summarizes the different routes to induce selfassembly and gelation of the nanoemulsion studied in this work. We start with the thermal gelation of the nanoemulsion via PEGDA bridging. Afterward, we study the effect of electrolyte screening on the material properties by adding $\mathrm{NaCl}$ into the nanoemulsion suspension. Finally, we study the material behavior by sequentially inducing the screening of electrostatic repulsion via the addition of $\mathrm{NaCl}$ and then the PEGDA bridging via the increase in temperature. The material behavior is characterized by rheometry and confocal microscopy. 
A

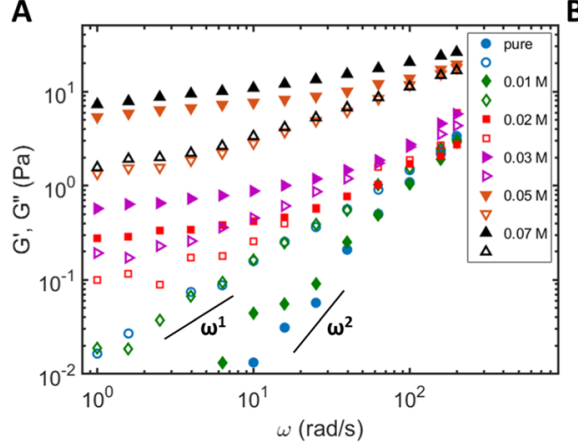

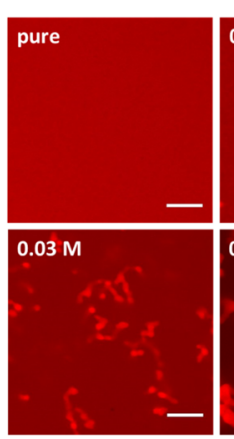
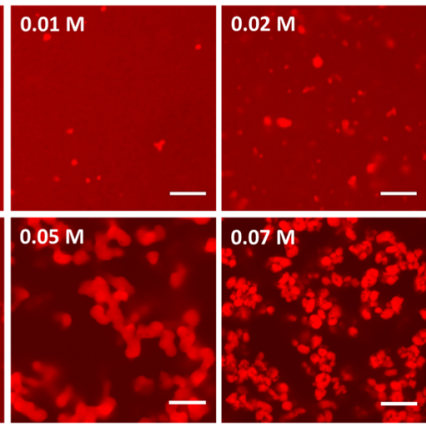

Figure 3. Rheological response and the associated microstructures of the nanoemulsion at various values of added salt $[\mathrm{NaCl}]$, at $\mathrm{T}=20.0{ }^{\circ} \mathrm{C}$. $(\mathrm{A})$ Linear viscoelastic moduli (closed symbols are storage moduli $G^{\prime}$, open symbols are loss moduli $G^{\prime \prime}$ ) as a function of angular frequency $\omega$. (B) Microstructure of the assembled nanoemulsions at various values of added salt $[\mathrm{NaCl}]$. Scale bars $=10 \mu \mathrm{m}$.

3.1. Thermally gelling Nanoemulsions via PEGDA Droplet Bridging. Here, we briefly introduce the thermallytriggered PEGDA droplet bridging and the associated nanoemulsion rheology and microstructures. Readers interested in more detailed studies of this thermally gelling nanoemulsion system are referred to the previous works by our group. ${ }^{28-32}$ As depicted in Figure 1, the thermally gelling nanoemulsion is a result of the inter-droplet bridging via PEGDA molecules. At elevated temperatures, the acrylate groups at the termini of PEGDA become increasingly hydrophobic and partition into the oil/water interface, leading to droplet bridging. ${ }^{29}$ The linear viscoelastic moduli (storage modulus $G^{\prime}$ and loss modulus $G^{\prime \prime}$ ) and the associated microstructures of the nanoemulsions at elevated temperatures are shown in Figure 2. At room temperature $\left(T=20.0^{\circ} \mathrm{C}\right)$, the nanoemulsion shows a liquid-like behavior in which $G^{\prime} \approx \omega^{2}$ and $G^{\prime \prime} \approx \omega^{1}$ (Figure $2 \mathrm{~A}$ ), ${ }^{39}$ and no assembled structure is observed by confocal microscopy (Figure 2B). Upon increasing the temperature, $G^{\prime}$ and $G^{\prime \prime}$ increase, and the system undergoes a sol-gel transition between $T=30.0{ }^{\circ} \mathrm{C}$ and $32.5^{\circ} \mathrm{C}$, upon which $G^{\prime}$ is greater than $G^{\prime \prime}$ and the system behaves as a viscoelastic solid (see more discussion on the critical gelation point in the Supporting Information). The increase in the mechanical strength results from the assembled gel network. As shown in Figure 2B, at elevated temperatures, the nanoemulsion droplets first assemble into cluster aggregates $\left(T=30.0{ }^{\circ} \mathrm{C}\right)$ and then a sparse gel network is formed $\left(T=32.5{ }^{\circ} \mathrm{C}\right) . .^{31,40}$ When the temperature is further increased, a more well-connected and space-filling network is formed. The gelling nanoemulsion shows a classic arrested phase separation behavior in which the microstructure shows the bicontinuous phases composed of droplet-rich domains (fluorescence region) and droplet-lean domains (dark regime), and the network is denser when the attraction is stronger (i.e., the temperature is higher in our system). ${ }^{41-43}$

3.2. Screening of Electrostatic Repulsion via $\mathrm{NaCl}$. For charge-stabilized colloidal suspensions, it is widely known that one can induce colloidal assembly and gelation by addition of an electrolyte to screen the interparticle electrostatic repulsion. ${ }^{26,27}$ As our nanoemulsion is stabilized by ionic surfactants (SDS), we expect that the same screening effect can take place in our system. Figure 3 shows the rheological response and the associated microstructure of the nanoemulsion at different concentrations of added $\mathrm{NaCl},[\mathrm{NaCl}]$, at $T=20.0^{\circ} \mathrm{C}$. As anticipated, the gelation of nanoemulsions can be successfully induced by increasing $[\mathrm{NaCl}]$ in the suspension. When $[\mathrm{NaCl}] \leq 0.01 \mathrm{M}$, the nanoemulsion shows a liquid-like behavior where $G^{\prime} \approx \omega^{2}$ and $G^{\prime \prime} \approx \omega^{1}$ (Figure 3A), although a few aggregates composed of nanoemulsion droplets are induced at $[\mathrm{NaCl}]=0.01 \mathrm{M}$. When $[\mathrm{NaCl}]$ is further increased, the viscoelastic moduli significantly increase and more aggregates are induced, which eventually span the space and give rise to a solid-like behavior of the material (see more discussion on the critical gelation point in the Supporting Information). Compared to the gelation induced by PEGDA bridging, the salt-induced gelation results in a weaker gel (Figure 2A vs Figure 3A) and a more open gel network (Figure 2B vs Figure 3B).

Interestingly, we note that the salt concentration needed to induce gelation is quite low in our system $([\mathrm{NaCl}] \approx 0.01-0.1$ $\mathrm{M})$, as compared to other investigations where SDS-stabilized PDMS nanoemulsions were also studied $([\mathrm{NaCl}] \approx 0.1-1$ M). ${ }^{14,25}$ Moreover, even a slight variation in $[\mathrm{NaCl}]$ can give rise to a significant change in the suspension rheology and associated microstructures. We proposed two effects that are responsible for such sensitivity to added salts. First, at room temperature where PEGDA droplet bridging is not induced, the nonassociating PEGDA acts as a depletant in the system that gives to an attractive interaction with a strength $=15.5 \mathrm{kT}$, where $k$ is Boltzmann constant and $T$ is the absolute temperature (the calculation will be discussed in detail in the next section). The addition of salt screens the electrostatic repulsion and then makes the strong depletion attraction easily dominate. Second, the length scales associated with the range of these interactions also play an important role. For electrostatic repulsion, the effective length scale is characterized by the Debye length, ${ }^{26,27} \kappa^{-1}$, and the results are shown in Table 1. Details of the calculation will be discussed in the next section. Because an excess of SDS is added to facilitate the nanoemulsion synthesis, there is already a large concentration of charged species in the continuous phase even without added $\mathrm{NaCl}$. Therefore, $\kappa^{-1}$ is $0.71 \mathrm{~nm}$ for $[\mathrm{NaCl}]=0 \mathrm{M}$. On the

Table 1. Dielectric Constant, $\boldsymbol{\kappa}^{-1}$, at Various Concentration of $\mathrm{NaCl},[\mathrm{NaCl}]$

$\begin{array}{cc}{[\mathrm{NaCl}](\mathrm{M})} & \kappa^{-1}(\mathrm{~nm}) \\ 0 & 0.71 \\ 0.01 & 0.68 \\ 0.02 & 0.66 \\ 0.03 & 0.64 \\ 0.05 & 0.60 \\ 0.07 & 0.57\end{array}$




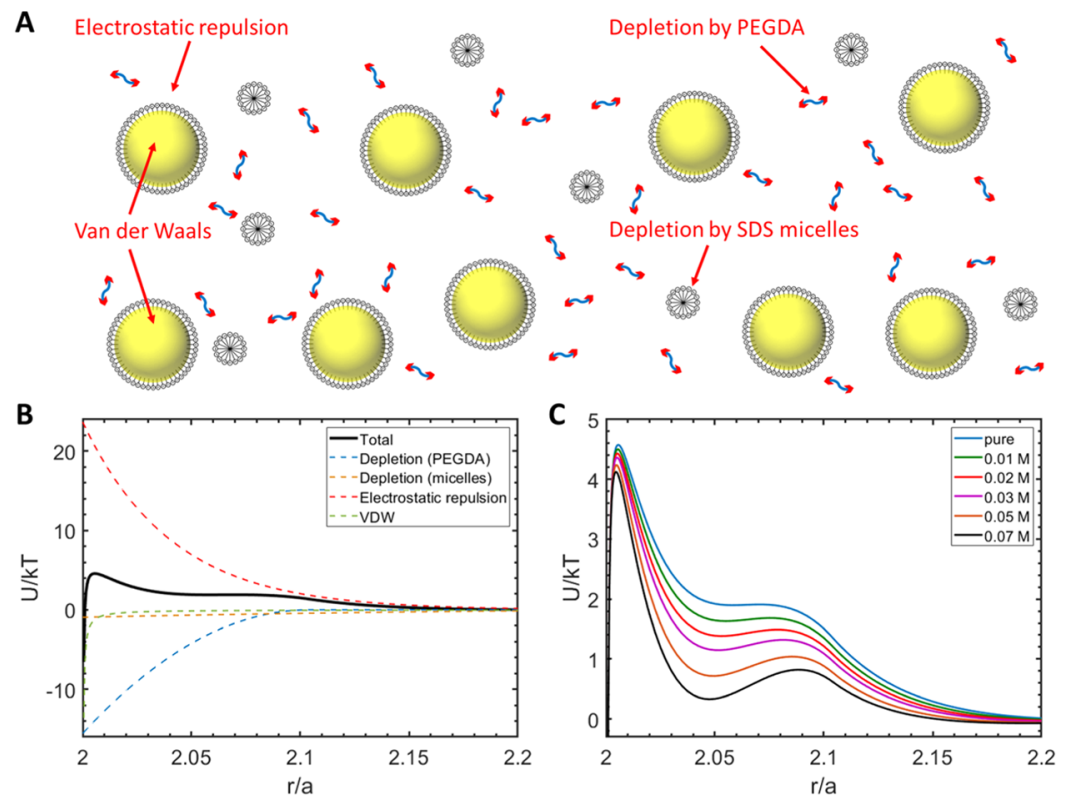

Figure 4. Estimates of interaction potentials at various $[\mathrm{NaCl}]$ of the nanoemulsion at $T=20^{\circ} \mathrm{C}$. (A) Schematic of the system used for estimating the interaction potentials. A total of four interactions were considered: screened electrostatic repulsions, depletion by PEGDA, depletion by SDS micelles, and VDW interaction. (B) Contributions to the potential at $[\mathrm{NaCl}]=0 \mathrm{M}$ and $\mathrm{T}=20^{\circ} \mathrm{C}$ from depletion by PEGDA, depletion by SDS micelles, electrostatic repulsion, and VDW interaction. (C) Overall interaction potential at various values of added $[\mathrm{NaCl}]$ at $\mathrm{T}=20{ }^{\circ} \mathrm{C}$.

other hand, for depletion interaction the range of the interaction is determined by the size of the depletant ${ }^{19}-$ here the radius gyration $\left(R_{\mathrm{g}}\right)$ of PEGDA is approximately 0.7 $\mathrm{nm} .{ }^{44}$ As can be seen, the range of the attractive and repulsive interactions are comparable, and a slight decrease in $\kappa^{-1}$ can easily make the depletion attraction dominant in the system.

3.3. Estimation of Interactive Potentials. To support the discussion in the last section, we present here a quantitative description of the interactions involved in the nanoemulsion system. We consider the scenario shown in Figure 4A where the system is composed of charged-stabilized nanoemulsion droplets, nonadsorbing PEGDA, SDS micelles, sodium ions, and chloride ions. The schematic diagram in Figure 4A then describes the scenario of the salt-added nanoemulsion at room temperature and with no PEGDA droplet bridging. Estimation of the pairwise interactive potentials follows the methodology developed in our prior work ${ }^{15}$ which has been shown to successfully describe the interactive potentials of a similar gelling nanoemulsion system. As shown in Figure 4A, the overall pairwise interaction between droplets has contributions from screened electrostatic repulsion, depletion attraction arising from both PEGDA molecules and SDS micelles in the continuous phase, and van der Waals (VDW) interaction. Next, we briefly summarize how to estimate each interaction.

3.4. Electrostatic Repulsion. The electrostatic repulsion, $U_{\text {elec }}$ is estimated using the Yukawa potential ${ }^{26,27}$

$$
U_{\text {elec }}=\varepsilon_{\text {elec }} \frac{2 a}{r} \mathrm{e}^{-\kappa(r-2 a)}
$$

where $a$ and $r$ are the radius and the center-to-center distance of nanoemulsion droplets, respectively. $\kappa^{-1}$ is the Debye length and is calculated as follows

$$
\kappa^{-1}=\sqrt{\frac{\varepsilon_{\mathrm{o}} \varepsilon_{\mathrm{r}} k T}{1000 e^{2} N_{\mathrm{A}} \sum z_{i}^{2} M_{i}}}
$$

where $\varepsilon_{\mathrm{o}}$ is the electric permeability of free space, $k$ is Boltzmann constant, $T$ is the absolute temperature, $e$ is the elementary charge, $N_{\mathrm{A}}$ is Avogadro's number, $z_{i}$ is the charge number ( $=1$ because both $\mathrm{NaCl}$ and SDS are monovalent), and $M_{i}$ is the molar concentration. $M_{i}$ is determined by the molar concentration of the added $\mathrm{NaCl}$ and the remaining free [SDS] in the continuous phase that is estimated by considering the area of SDS occupied on the oil droplet $\left(=0.617 \mathrm{~nm}^{2}\right)$ molecule) ${ }^{45} \varepsilon_{\mathrm{r}}$ is the dielectric constant of the continuous phase, which is calculated by considering the dielectric constants of water ${ }^{46}$ and PEGDA. The dielectric constant of PEGDA is estimated from the dielectric constant of the PEG backbone $\left(\varepsilon_{\mathrm{r}, \mathrm{PEG}}=14.5\right){ }^{47} \varepsilon_{\mathrm{r}}$ is then calculated using the mixing rule, for which the deviation from the measured value has been shown to be negligible at the high PEG concentration regime such as the case in our system. ${ }^{48}$

The strength of the electrostatic repulsion, $\varepsilon_{\text {elec }}$ is calculated using the following equation ${ }^{26,27}$

$$
\varepsilon_{\text {elec }}=32 \pi \varepsilon_{\mathrm{o}} \varepsilon_{\mathrm{r}}\left(\frac{k T}{z e}\right)^{2} a \tan h^{2}\left(\frac{1}{4} \frac{z e \xi}{k T}\right)
$$

which is obtained from Gouy-Chapman solution to PoissonBoltzmann equation with the superposition assumption and Derjaguin approximation. The zeta potential, $\xi$, of the nanoemulsion droplets is $-44.3 \mathrm{mV}$ and is measured using a Zetasizer (Brookhaven Instruments 90Plus PALS).

3.5. PEGDA Depletion. The PEGDA depletion is estimated from the Asakura-Oosawa potential ${ }^{19,20}$

$$
U_{\mathrm{dep}}=-\frac{\varepsilon_{\mathrm{dep}}\left(a+\delta_{\mathrm{P}}\right)^{3}}{\delta_{\mathrm{p}}{ }^{2}\left(\frac{3 a}{2}+\delta_{\mathrm{p}}\right)}\left[1-\frac{3}{4} \frac{r}{a+\delta_{\mathrm{P}}}+\frac{1}{16}\left(\frac{r}{a+\delta_{\mathrm{P}}}\right)^{3}\right]
$$

when $2 a \leq r \leq 2\left(a+\delta_{\mathrm{p}}\right)$. To consider the polymeric nature of PEGDA (instead of simple hard-spheres), we use the ideal polymer assumption. Therefore, an additional configurational 

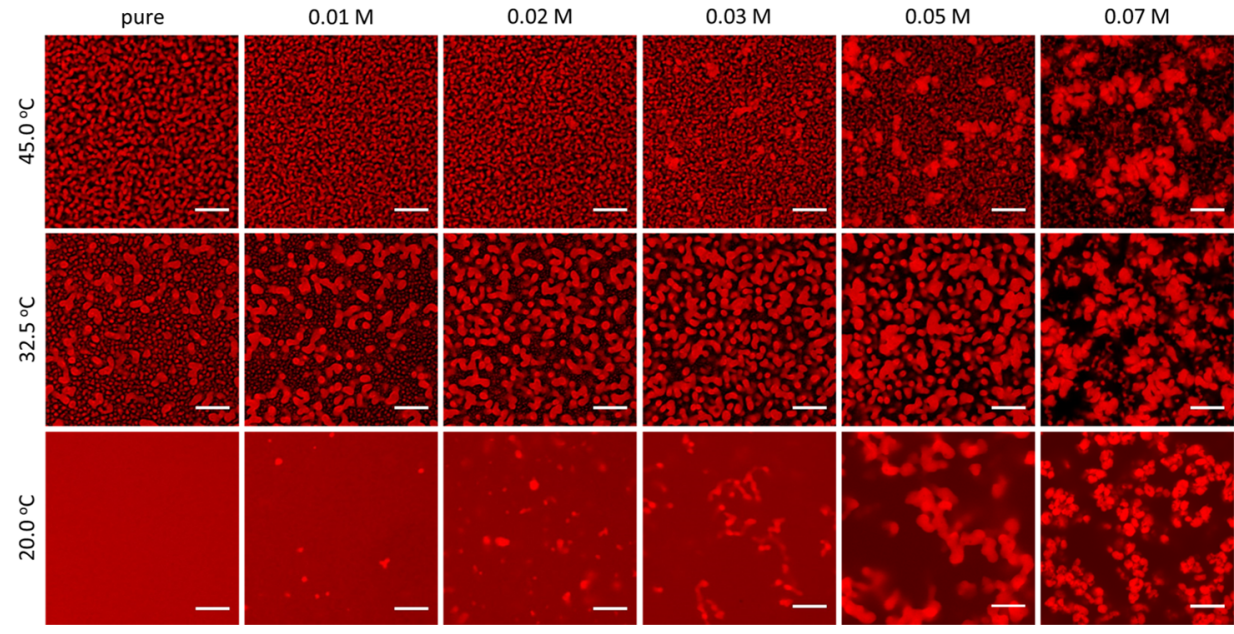

Figure 5. Microstructures of the nanoemulsion observed after sequentially screening the electrostatic repulsion, and then inducing PEGDA thermal bridging of droplets. Scale bars $=10 \mu \mathrm{m}$.

entropy from the polymeric depletants contributes to the depletion strength, $\varepsilon_{\text {dep }}$, and the interaction range, $\delta_{\mathrm{P} .}{ }^{19} \varepsilon_{\text {dep }}$ and $\delta_{\mathrm{p}}$ are then estimated as

$$
\begin{aligned}
& \varepsilon_{\mathrm{dep}}=\phi_{\mathrm{p}} \frac{a}{R_{\mathrm{g}}} 3 \ln 2 \\
& \delta=\sqrt{2 \ln 2} R_{\mathrm{g}}
\end{aligned}
$$

where $\phi_{\mathrm{p}}$ is the PEGDA volume fraction and $R_{\mathrm{g}}$ is the radius of gyration of PEGDA $(\approx 0.7 \mathrm{~nm}) .{ }^{44}$ For the estimation of the potential, we assumed that the morphology of PEGDA is independent of the $[\mathrm{NaCl}]$. If no salt is added, considering the length of PEGDA $\left(M_{\mathrm{n}} \approx 700 \mathrm{~g} \mathrm{~mol}^{-1}\right)$ and the experimental temperature window, water is a good solvent. ${ }^{49-52}$ On the other hand, the change in the ionic strength does affect the affinity between PEGs and water. It has been shown that an increase in salt concentration makes water a poorer solvent to PEGs. ${ }^{52-54}$ However, to induce such change in affinity between PEG and water, the concentration of salt should be at least on the order of $0.1 \mathrm{M}$. However, for our study the concentration of salt that we add into the nanoemulsion system is $\sim 0.01 \mathrm{M}$. Therefore, we believe that the amount of salt we add into the nanoemulsions system has a negligible effect on $R_{\mathrm{g}}$ and water is still a good solvent across the experimental $[\mathrm{NaCl}]$ window. Therefore, in our calculations, we assume that PEGDA depletion remains constant for all values of $[\mathrm{NaCl}]$.

3.6. SDS Micelle Depletion. Depletion by SDS micelles is also estimated by the Asakura-Oosawa potential. Assuming micelles behave like hard-spheres, the micelle depletion is calculated as follows ${ }^{19,22,55}$

$$
U_{\mathrm{dep}}=-\left(\xi C_{\mathrm{m}} k T\right)\left(\frac{\pi a}{2}\left(d_{\mathrm{m}, \mathrm{eff}}-(r-2 a)\right)^{2}\right)
$$

when $2 a \leq r \leq 2 a+d_{\mathrm{m}, \mathrm{eff}}$ and

$$
\xi=\frac{1+\phi_{\mathrm{m}, \mathrm{eff}}+\phi_{\mathrm{m}, \mathrm{eff}}{ }^{2}-\phi_{\mathrm{m}, \mathrm{eff}}{ }^{3}}{\left(1-\phi_{\mathrm{m}, \mathrm{eff}}\right)^{3}}
$$

where $\phi_{\mathrm{m} \text {,eff }}$ is the effective volume fraction of micelles by considering the Debye layer ${ }^{36}$ and is calculated as

$$
\phi_{\mathrm{m}, \mathrm{eff}}=\frac{4 \pi}{3}\left(\frac{d_{\mathrm{m}, \mathrm{eff}}}{2}\right)^{3} C_{\mathrm{m}}=\frac{4 \pi}{3}\left(\frac{d_{\mathrm{m}}+2 \kappa^{-1}}{2}\right)^{3} C_{\mathrm{m}}
$$

$C_{\mathrm{m}}$ is the concentration of micelles and is calculated as

$$
C_{\mathrm{m}}=\frac{\left(C_{\mathrm{o}}-C_{\mathrm{ads}}-C_{\mathrm{CMC}}\right) N_{\mathrm{A}}}{N_{\mathrm{agg}}}
$$

where $C_{\mathrm{o}}$ is the total concentration of SDS, $C_{\mathrm{ads}}$ is the concentration of SDS adsorbed on the droplets for stabilization by considering the area of SDS occupied on the oil droplet (0.617 $\mathrm{nm}^{2} /$ molecule $),{ }^{45} C_{\mathrm{CMC}}$ is the critical micelle concentration $(\approx 8 \mathrm{mM}),{ }^{56-58}$ and $N_{\text {agg }}$ is the micelle aggregation number $(\approx 60) .^{56-58}$ For the estimation, we assume that the morphology of the micelles is independent of $[\mathrm{NaCl}]$.

3.7. VDW Interaction. VDW interaction is estimated as follows $^{27}$

$$
U_{\mathrm{VDW}}=\frac{-A a}{12(r-2 a)}
$$

by assuming a uniform droplet size and the Derjaguin approximation is used. Here, $A=3.3 \times 10^{-22} \mathrm{~J}$ is the Hamaker constant of PDMS-water-PDMS. ${ }^{59,60}$

3.8. Overall Pairwise Interaction Potentials. Figure $4 \mathrm{~B}$ shows the resulting contribution from each interaction to the overall interaction at $T=20.0^{\circ} \mathrm{C}$ and $[\mathrm{NaCl}]=0 \mathrm{M}$. In this system, the repulsion comes from the electrostatic repulsion provided by the charged SDS on the nanoemulsion droplets, and the major attraction comes from the PEGDA depletion via the free PEGDA in the continuous phase. Although PEGDA provides a significant depletion attraction with a strength = $15.5 \mathrm{kT}$, the even stronger electrostatic repulsion dominates the interaction. Therefore, no minimum in the overall pairwise interaction is observed and no self-assembly is induced. Although the electrostatic repulsion prevails in the system at $T=20.0^{\circ} \mathrm{C}$ and $[\mathrm{NaCl}]=0 \mathrm{M}$, the addition of electrolytes can effectively screen the electrostatic repulsion by decreasing its characteristic length scale (i.e., Debye length, $\kappa^{-1}$ ), leading to a local minimum in the interactive potential. Figure $4 \mathrm{C}$ shows the result of the interactive potential at increasing $[\mathrm{NaCl}]$ at $T$ $=20.0{ }^{\circ} \mathrm{C}$. As can be observed, a local minimum is induced when $\mathrm{NaCl}$ is added, and the depth of the minimum is greater 
when more $\mathrm{NaCl}$ is added. Moreover, the overall pairwise interaction captures the material property trends of the nanoemulsions seen in Figure 3. When the ionic strength increases for $[\mathrm{NaCl}] \leq 0.02 \mathrm{M}$, a shallow minimum emerges, and few small aggregates are observed in the system. As the ionic strength is further increased for $[\mathrm{NaCl}] \geq 0.03 \mathrm{M}$, larger aggregates are formed and eventually a spanning network is established, and the nanoemulsion turns into a viscoelastic solid (Figure 3A).

\subsection{Sequentially Triggering Electrostatic Screening} and Then PEGDA Bridging. Having established a quantitative understanding of nanoemulsion behavior under the effect of electrolyte screening, we now sequentially trigger the PEGDA droplet bridging by increasing the temperature of the system, as depicted by the bottom route in Figure 1 .

Figure 5 shows the microstructures resulting from this sequential assembly process. Again, in this work, we first increase the ionic strength of the system at $T=20.0{ }^{\circ} \mathrm{C}$ (as shown in the bottom row of Figure 5) and then increase the temperature to induce the PEGDA droplet bridging (moving vertically upward from the bottom of Figure 5). We chose the temperatures for these studies to be 32.5 and $45.0{ }^{\circ} \mathrm{C}$ to represent the point near (right above) the gelation point of the pure system $([\mathrm{NaCl}=0 \mathrm{M})$ and the point where the gel network is well-established due to the thermal PEGDA droplet bridging, respectively. Generally, as more $\mathrm{NaCl}$ is added, at elevated temperatures there are more remnants from the saltinduced structure at $T=20.0{ }^{\circ} \mathrm{C}$, even though stronger PEGDA droplet bridging is induced at $T=45.0^{\circ} \mathrm{C}$. In other words, when $[\mathrm{NaCl}]$ is higher at $T=20.0{ }^{\circ} \mathrm{C}$, the nanoemulsion has more difficulty rearranging its assembled structures at this low temperature via subsequent thermally induced PEGDA bridging.

For $[\mathrm{NaCl}] \leq 0.02 \mathrm{M}$, the microstructures across the experimental $[\mathrm{NaCl}]$ window are similar at $T=45.0{ }^{\circ} \mathrm{C}$ regardless of $[\mathrm{NaCl}]$. However, the nanoemulsions with added salt display a more established network of thick strands at $T=$ $32.5{ }^{\circ} \mathrm{C}$. Trends in the microstructures can be quantitatively analyzed by calculating the characteristic length, $L_{C}$, of the structure by applying a fast Fourier transform to the confocal images and then calculating the correlation length from the scattering spectrum (see the Supporting Information, Figure S5 for an example of the calculation). ${ }^{30-33,43}$ The results for the calculated $L_{\mathrm{C}}$ are shown in Figure 6. For $[\mathrm{NaCl}] \leq 0.02 \mathrm{M}$, $L_{\mathrm{C}}$ at $T=32.5{ }^{\circ} \mathrm{C}$ decreases with increasing $[\mathrm{NaCl}]$, corresponding to the increase in the number of thick strands

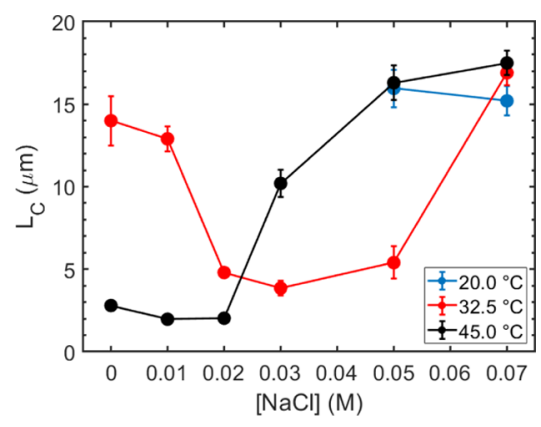

Figure 6. Correlation length, $L_{C}$, calculated from images in Figure 5 as a function of $[\mathrm{NaCl}]$ for three different temperatures. $L_{\mathrm{C}}$ of images for $[\mathrm{NaCl}] \leq 0.03 \mathrm{M}$ at $T=20^{\circ} \mathrm{C}$ cannot be characterized. Error bars are standard errors from 20 images. seen in the confocal images. On the other hand, at $T=45.0$ ${ }^{\circ} \mathrm{C}, L_{\mathrm{C}}$ stays nearly constant over this $[\mathrm{NaCl}]$ window, suggesting that a similar gel network is obtained. However, for $[\mathrm{NaCl}] \geq 0.03 \mathrm{M}$, more remnants from the salt-induced structure at $T=20.0{ }^{\circ} \mathrm{C}$ are observed at elevated temperatures. At $T=32.5$ and $45.0^{\circ} \mathrm{C}$, it can be easily observed that there are large aggregates incorporated into the gel network, and the number and the size of the aggregates are larger when $[\mathrm{NaCl}]$ is higher. These trends are also observed in Figure 6 for $[\mathrm{NaCl}] \geq 0.03 \mathrm{M}$, where $L_{\mathrm{C}}$ increases as $[\mathrm{NaCl}]$ is increased at $T=32.5$ and $45.0^{\circ} \mathrm{C}$.

The viscoelastic moduli under each condition are also measured, and the results are shown in Figure 7. Interestingly, the rheological data shows the trend that a stronger gel is not necessarily obtained with added $\mathrm{NaCl}$ even though the gelation is "pre-induced" with the addition of $\mathrm{NaCl}$ at room temperature (in this work, we use $G^{\prime}$ values to describe the relative strength of gels). This nonintuitive trend can be more clearly seen in Figure 8 where the $G^{\prime}$ at an intermediate angular frequency $\left(\omega=10 \mathrm{rad} \mathrm{s}^{-1}\right)$ from Figure 7 is plotted as a function of $[\mathrm{NaCl}]$ for the three temperatures. For $T=20.0$ ${ }^{\circ} \mathrm{C}$ (no PEGDA droplet bridging is induced), the gel is stronger when more $\mathrm{NaCl}$ is added. However, when the temperature is increased to $T=45.0{ }^{\circ} \mathrm{C}$, the gel is weaker when more $\mathrm{NaCl}$ is added at $\mathrm{T}=20.0{ }^{\circ} \mathrm{C}$, even though the system starts with a "pre-gel" state with non-negligible mechanical strength. For $T=32.5{ }^{\circ} \mathrm{C}$, the gel strength increases first when the nanoemulsion starts with a low concentration of $\mathrm{NaCl}$ for $[\mathrm{NaCl}] \leq 0.03 \mathrm{M}$, and $G^{\prime}$ decreases when $[\mathrm{NaCl}]$ is further increased.

We believe two reasons are responsible for this nonintuitive trend in the microstructures and the rheological response. The first reason is related to the depth of the secondary minimum induced by the increased $[\mathrm{NaCl}]$ at $\mathrm{T}=20.0{ }^{\circ} \mathrm{C}$ (Figure $4 \mathrm{C}$ ). When $[\mathrm{NaCl}]$ is higher, the secondary minimum is deeper. For the structure to be rearranged as the temperature is increased, the system needs to escape from this minimum and undergo the PEGDA droplet bridging. Therefore, it is reasonable that with increasing $[\mathrm{NaCl}]$, there are more remnants of the saltinduced structures at $T=20.0{ }^{\circ} \mathrm{C}$ observed at the higher temperatures (Figure 5). The same mechanism applies to the rheological behavior in Figure 8. In the analysis in Section 3.3 and Figure 4 where the effect of $\mathrm{NaCl}$ screening at $\mathrm{T}=20.0^{\circ} \mathrm{C}$ is discussed, it is shown that the PEGDA depletion plays an important role. At $T=20.0^{\circ} \mathrm{C}$, PEGDA droplet bridging is not induced and these nonadsorbing PEGDA polymers behave as depletants in the system. Because the depletion is a result of increased osmotic pressure, ${ }^{19}$ the depletants are excluded from inside the aggregated colloids when the clusters and gel network are formed. ${ }^{1}$ In our study, the PEGDA plays the role of both depletant and thermally triggered droplet bridging agent (at $T=32.5$ and $45.0{ }^{\circ} \mathrm{C}$ ). When the salt-induced structures are more difficult to be reconstructed (at increased $[\mathrm{NaCl}]$ ), PEGDA cannot easily enter between droplets and undergo bridging. Moreover, from the previous discussion in Sections 3.1 and 3.2 as well as in Figure 8, it can be observed that a weak gel is obtained by pure electrolyte screening (blue data in Figure 8 at $T=20.0{ }^{\circ} \mathrm{C}$ ) compared to the pure thermally bridging (red and black data in Figure 8 at $[\mathrm{NaCl}]=$ $0 \mathrm{M})$. Therefore, when $[\mathrm{NaCl}]$ is high, the salt-induced aggregation is strong enough to stay intact when the temperature is increased. Under these conditions, the system has difficulty rearranging, the effect of PEGDA bridging is 

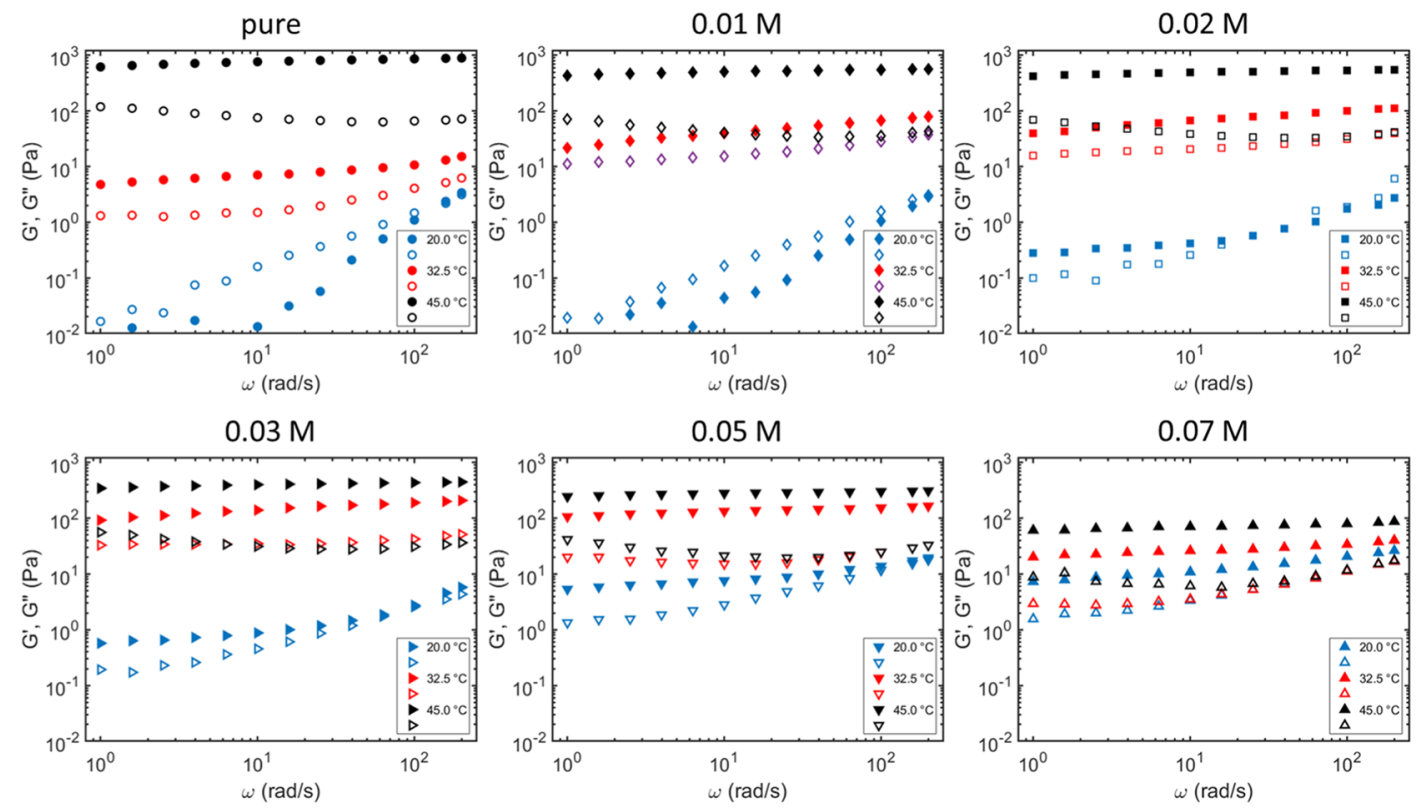

Figure 7. Linear viscoelastic moduli (closed symbols $=G^{\prime}$, open symbols $=G^{\prime \prime}$ ) as a function of angular frequency, $\omega$, of the nanoemulsion after sequentially screening the electrostatic repulsion via added salt, and then thermally induced bridging of droplet by PEGDA.

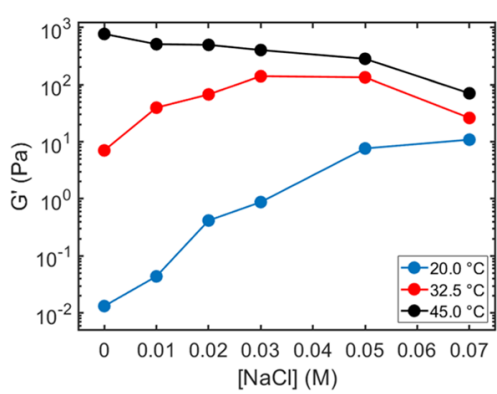

Figure 8. Storage modulus, $G^{\prime}$ (at $\omega=10 \mathrm{rad} \mathrm{s}^{-1}$ ), as a function of $[\mathrm{NaCl}]$ for three temperatures. Data is from Figure 7.

diminished, and hence a weaker gel results (black data in Figure 8).

The second reason explaining the trends in microstructures and rheology relates to the dynamics of the PEGDA bridginginduced gelation. It can be noticed that the first reason as just discussed (the depth of the minimum in the pairwise interactive potentials and the mechanism of PEGDA depletion and bridging) describes well the material behavior at $T=45.0$ ${ }^{\circ} \mathrm{C}$ (and at $\mathrm{T}=32.5{ }^{\circ} \mathrm{C}$ for $[\mathrm{NaCl}]>0.03 \mathrm{M}$ ), where both microstructure and rheology have a monotonic trend $-L_{\mathrm{C}}$ monotonically increases (Figure 6) and $G^{\prime}$ monotonically decreases (Figure 8) when the nanoemulsion is preconditioned with higher $[\mathrm{NaCl}]$. However, for $\mathrm{T}=32.5{ }^{\circ} \mathrm{C}$, a nonmonotonic trend is observed; $L_{C}$ first decreases, then increases and $G^{\prime}$ first increases and then decreases when the nanoemulsion is preconditioned with higher $[\mathrm{NaCl}]$. We believe this is due to the dynamics of the gelation via PEGDA bridging. In our prior works, we have shown that under the thermally triggered PEGDA bridging, the gelation of nanoemulsions undergoes a spinodal decomposition and then the structure is dynamically arrested. ${ }^{30,32}$ Under this gelation route, a stronger attraction (i.e., PEGDA bridging at higher temperatures) gives rise to an earlier arrest and stops the network coarsening. ${ }^{61-63}$ This behavior can be characterized by measuring the viscoelastic moduli with time at different temperatures (Figure S6), where at $T=45.0{ }^{\circ} \mathrm{C}$, the moduli reach a plateau after $t \approx 400 \mathrm{~s}$, whereas at $T=32.5{ }^{\circ} \mathrm{C}$, the moduli still increase with time over the experimental window $(t=600 \mathrm{~s})$. In other words, the large-scale structural reconstruction of the salt-induced structure is suppressed at high temperatures, as the strong PEGDA bridging can more effectively dynamically arrest the gel network, whereas at lower temperatures, the system can more effectively take advantage of the "pre-gel" structures induced by the added salt and undergo structural reconstruction. Therefore, compared to the nanoemulsion at $\mathrm{T}=45.0{ }^{\circ} \mathrm{C}$, the rearrangement is more prominent for $T=32.5{ }^{\circ} \mathrm{C}$ for $[\mathrm{NaCl}] \leq 0.03 \mathrm{M}$ as $L_{\mathrm{C}}$ decreases (Figure 6) and $G^{\prime}$ increases (Figure 8) with $[\mathrm{NaCl}]$. Such rearrangement can be also visually observed from the microstructures shown in Figure 5. At $T=32.5{ }^{\circ} \mathrm{C}$, the gel network coexists with freely-suspending clusters. We have shown in our prior work that such coexistent structures are a characteristic of the thermally gelling nanoemulsion near the critical gelation temperature. ${ }^{31,32}$ This observation serves as a strong evidence that the PEGDA bridging-induced reconstruction takes place, and the formation of clusters can be used as a qualitative scale to evaluate how effectively the thermally triggered PEGDA bridging rearranges the assembled network. As more $[\mathrm{NaCl}]$ is added at $\mathrm{T}=20.0{ }^{\circ} \mathrm{C}$, fewer clusters are observed and a more well-connected gel network is formed. When $[\mathrm{NaCl}]$ is above $0.03 \mathrm{M}$, the coexisting clusters at $T=$ $32.5{ }^{\circ} \mathrm{C}$ cannot be observed, and the trend of material properties at $T=32.5{ }^{\circ} \mathrm{C}$ follows the trend of those at $T=45.0$ ${ }^{\circ} \mathrm{C}$, and again we believe that it is because the depth of the minimum is too deep for the system to reconstruct itself, as we described earlier.

\section{CONCLUSIONS}

In this work, we introduce an approach to modulate the microstructures and the rheological properties of a nanoemulsion suspension by sequentially screening the electrostatic repulsion and then thermally triggering the droplet bridging. We revisited the nanoemulsion system previously developed in 
our group and carefully studied the inter-droplet interaction from each constituent in the system. By calculating the pairwise interactions, we found that the nonassociating PEGDA contributes to a significant depletion interaction, and the nanoemulsion system can undergo a sol-gel transition at room temperature by screening the electrostatic repulsion with the addition of $\mathrm{NaCl}$. By subsequently inducing the PEGDA droplet bridging via an increase in temperature, we show that the system can restructure and reach a different gelled state. We then used the established pairwise interactive potential and the dynamics of the thermal gelation to explain the trend in material behavior under the sequential application of stimuli. Our results suggest that the initial screening of the electrostatic repulsion can be used as an effective strategy to template the structure of the nanoemulsion system and the subsequent thermally bridging can then consolidate the gel network. Moreover, our discussion highlights that a quantitative understanding of the inter-droplet interaction is required to understand the behavior of the system. Future work will focus on establishing the pairwise interaction of the thermally bridging mechanism from first principles and applying simulations to better understand the system on the colloidal scale and the associated dynamics. Overall, this work provides molecular-level guidance to the design of colloid-based materials with complex microstructures by sequentially applying stimuli to the system.

\section{ASSOCIATED CONTENT}

\section{SI Supporting Information}

The Supporting Information is available free of charge at https://pubs.acs.org/doi/10.1021/acs.langmuir.0c00199.

Flow curve of the nanoemulsion gel from screening of electrostatic repulsion, shear stress versus shear rate of the nanoemulsion gel, linear viscoelastic moduli as a function of time, discussion of critical gelation point, and $L_{\mathrm{C}}$ calculation using the image processing software ImageJ (PDF)

\section{AUTHOR INFORMATION}

\section{Corresponding Author}

Patrick S. Doyle - Department of Chemical Engineering, Massachusetts Institute of Technology, Cambridge, Massachusetts 02139, United States; 이이이.org/0000-00032147-9172; Email: pdoyle@mit.edu

\section{Authors}

Li-Chiun Cheng - Department of Chemical Engineering, Massachusetts Institute of Technology, Cambridge, Massachusetts 02139, United States; 이이.org/0000-00015393-1982

Signe Lin Kuei Vehusheia - Department of Materials, ETH Zürich, 8093 Zürich, Switzerland

Complete contact information is available at: https://pubs.acs.org/10.1021/acs.langmuir.0c00199

\section{Notes}

The authors declare no competing financial interest.

\section{ACKNOWLEDGMENTS}

This work was supported by the MRSEC Program of the National Science Foundation under award number DMR-
1419807 and the National Science Foundation grant CMMI1824297.

\section{REFERENCES}

(1) Zaccarelli, E. Colloidal gels: equilibrium and non-equilibrium routes. J. Phys. Condens. Matter 2007, 19, 323101-323150.

(2) Hsiao, L. C.; Pradeep, S. Experimental synthesis and characterization of rough particles for colloidal and granular rheology. Curr. Opin. Colloid Interface Sci. 2019, 43, 94-112.

(3) Vintiloiu, A.; Leroux, J.-C. Organogels and their use in drug delivery - A review. J. Controlled Release 2008, 125, 179-192.

(4) Wang, Q.; Wang, J.; Lu, Q.; Detamore, M. S.; Berkland, C. Injectable PLGA based colloidal gels for zero-order dexamethasone release in cranial defects. Biomaterials 2010, 31, 4980-4986.

(5) He, C.; Kim, S. W.; Lee, D. S. In situ gelling stimuli-sensitive block copolymer hydrogels for drug delivery. J. Controlled Release 2008, 127, 189-207.

(6) Dickinson, E. Stabilising emulsion-based colloidal structures with mixed food ingredients. J. Sci. Food Agric. 2013, 93, 710-721.

(7) Dickinson, E. Colloids in Food: Ingredients, Structure, and Stability. Annu. Rev. Food Sci. Technol. 2015, 6, 211-233.

(8) Gan, T.; Zhang, Y.; Guan, Y. In Situ Gelation of P(NIPAMHEMA) Microgel Dispersion and Its Applications as Injectable 3D Cell Scaffold. Biomacromolecules 2009, 10, 1410-1415.

(9) Wang, Q.; Wang, L.; Detamore, M. S.; Berkland, C. Biodegradable colloidal gels as moldable tissue engineering scaffolds. Adv. Mater. 2008, 20, 236-239.

(10) Schmidt, D. F.; Du Fresne Von Hohenesche, C.; Weiss, A.; Schädler, V. Colloidal gelation as a general approach to the production of porous materials. Chem. Mater. 2008, 20, 2851-2853.

(11) Gupta, A.; Eral, H. B.; Hatton, T. A.; Doyle, P. S. Nanoemulsions: formation, properties and applications. Soft Matter 2016, 12, 2826-2841.

(12) Helgeson, M. E. Colloidal behavior of nanoemulsions: Interactions, structure, and rheology. Curr. Opin. Colloid Interface Sci. 2016, 25, 39-50.

(13) Kim, H. S.; Mason, T. G. Advances and challenges in the rheology of concentrated emulsions and nanoemulsions. Adv. Colloid Interface Sci. 2017, 247, 397-412.

(14) Fryd, M. M.; Mason, T. G. Advanced Nanoemulsions. Annu. Rev. Phys. Chem. 2012, 63, 493-518.

(15) Cheng, L.-C.; Sherman, Z. M.; Swan, J. W.; Doyle, P. S. Colloidal Gelation through Thermally Triggered Surfactant Displacement. Langmuir 2019, 35, 9464-9473.

(16) Sonneville-Aubrun, O.; Simonnet, J.-T.; L'Alloret, F. Nanoemulsions: a new vehicle for skincare products. Adv. Colloid Interface Sci. 2004, 108-109, 145-149.

(17) Yukuyama, M. N.; Ghisleni, D. D. M.; Pinto, T. J. A.; BouChacra, N. A. Nanoemulsion: process selection and application in cosmetics - a review. Int. J. Cosmet. Sci. 2016, 38, 13-24.

(18) Hsiao, L. C.; Badruddoza, A. Z. M.; Cheng, L.-C.; Doyle, P. S. 3D Printing of Self-Assembling Thermoresponsive Nanoemulsions into Hierarchical Mesostructured Hydrogels. Soft Matter 2017, 13, 921-929.

(19) Lekkerkerker, H. N. W.; Tuinier, R. Colloids and the Depletion Interaction; Springer: Netherlands, 2011.

(20) Asakura, S.; Oosawa, F. Interaction between particles suspended in solutions of macromolecules. J. Polym. Sci. 1958, 33, $183-192$.

(21) Asakura, S.; Oosawa, F. On interaction between two bodies immersed in a solution of macromolecules. J. Chem. Phys. 1954, 22, $1255-1256$

(22) Erramreddy, V. V.; Ghosh, S. Influence of emulsifier concentration on nanoemulsion gelation. Langmuir 2014, 30, $11062-11074$.

(23) Erramreddy, V. V.; Ghosh, S. Influence of droplet size on repulsive and attractive nanoemulsion gelation. Colloid. Surface. Physicochem. Eng. Aspect. 2015, 484, 144-152. 
(24) Wilking, J. N.; Graves, S. M.; Chang, C. B.; Meleson, K.; Lin, M. Y.; Mason, T. G. Dense Cluster Formation during Aggregation and Gelation of Attractive Slippery Nanoemulsion Droplets. Phys. Rev. Lett. 2006, 96, 015501.

(25) Wilking, J. N.; Chang, C. B.; Fryd, M. M.; Porcar, L.; Mason, T. G. Shear-Induced Disruption of Dense Nanoemulsion Gels. Langmuir 2011, 27, 5204-5210.

(26) Russel, W. B.; Saville, D. A.; Schowalter, W. R. Colloidal Dispersions; Cambridge University Press: Cambridge, 1989.

(27) Hiemenz, P. C.; Rajagopalan, R. Principles of Colloid and Surface Chemistry; CRC Press, 1997.

(28) Helgeson, M. E.; Gao, Y.; Moran, S. E.; Lee, J.; Godfrin, M.; Tripathi, A.; Bose, A.; Doyle, P. S. Homogeneous percolation versus arrested phase separation in attractively-driven nanoemulsion colloidal gels. Soft Matter 2014, 10, 3122-3133.

(29) Helgeson, M. E.; Moran, S. E.; An, H. Z.; Doyle, P. S. Mesoporous organohydrogels from thermogelling photocrosslinkable nanoemulsions. Nat. Mater. 2012, 11, 344-352.

(30) Hsiao, L. C.; Doyle, P. S. Celebrating Soft Matter's 10th Anniversary: Sequential phase transitions in thermoresponsive nanoemulsions. Soft Matter 2015, 11, 8426-8431.

(31) Cheng, L.-C.; Hsiao, L. C.; Doyle, P. S. Multiple particle tracking study of thermally-gelling nanoemulsions. Soft Matter 2017, 13, 6606-6619.

(32) Cheng, L.-C.; Godfrin, P. D.; Swan, J. W.; Doyle, P. S. Thermal processing of thermogelling nanoemulsions as a route to tune material properties. Soft Matter 2018, 14, 5604-5614.

(33) Gao, Y.; Kim, J.; Helgeson, M. E. Microdynamics and arrest of coarsening during spinodal decomposition in thermoreversible colloidal gels. Soft Matter 2015, 11, 6360-6370.

(34) Kim, J.; Merger, D.; Wilhelm, M.; Helgeson, M. E. Microstructure and nonlinear signatures of yielding in a heterogeneous colloidal gel under large amplitude oscillatory shear. J. Rheol. 2014, 58, 1359-1390.

(35) Bibette, J.; Mason, T. G.; Gang, H.; Weitz, D. A.; Poulin, P. Structure of adhesive emulsions. Langmuir 1993, 9, 3352-3356.

(36) Mason, T. G.; Wilking, J. N.; Meleson, K.; Chang, C. B.; Graves, S. M. Nanoemulsions: formation, structure, and physical properties. J. Phys. Condens. Matter 2006, 18, R635-R666.

(37) Lee, J. C.-W.; Porcar, L.; Rogers, S. A. Unveiling Temporal Nonlinear Structure-Rheology Relationships under Dynamic Shearing. Polymers 2019, 11, 1189.

(38) Lee, J. C.-W.; Weigandt, K. M.; Kelley, E. G.; Rogers, S. A. Structure-Property Relationships via Recovery Rheology in Viscoelastic Materials. Phys. Rev. Lett. 2019, 122, 248003.

(39) Mewis, J.; Wagner, N. J.Colloidal Suspension Rheology; Cambridge University Press: Cambridge, 2011.

(40) Cheng, L.-C.; Hashemnejad, S. M.; Zarket, B.; Muthukrishnan, S.; Doyle, P. S. Thermally and $\mathrm{pH}$-responsive gelation of nanoemulsions stabilized by weak acid surfactants. J. Colloid Interface Sci. 2020, 563, 229-240.

(41) Hsiao, L. C.; Newman, R. S.; Glotzer, S. C.; Solomon, M. J. Role of isostaticity and load-bearing microstructure in the elasticity of yielded colloidal gels. Proc. Natl. Acad. Sci. U.S.A. 2012, 109, 1602916034.

(42) Whitaker, K. A.; Varga, Z.; Hsiao, L. C.; Solomon, M. J.; Swan, J. W.; Furst, E. M. Colloidal gel elasticity arises from the packing of locally glassy clusters. Nat. Commun. 2019, 10, 2237.

(43) Zia, R. N.; Landrum, B. J.; Russel, W. B. A micro-mechanical study of coarsening and rheology of colloidal gels: Cage building, cage hopping, and Smoluchowski's ratchet. J. Rheol. 2014, 58, 11211157.

(44) Lee, H.; Venable, R. M.; MacKerell, A. D.; Pastor, R. W. Molecular dynamics studies of polyethylene oxide and polyethylene glycol: Hydrodynamic radius and shape anisotropy. Biophys. J. 2008, 95, 1590-1599.

(45) Kanellopoulos, A. G.; Owen, M. J. Adsorption of sodium dodecyl sulphate at the silicone fluid/water interface. Trans. Faraday Soc. 1971, 67, 3127-3138.
(46) Malmberg, C. G.; Maryott, A. A. Dielectric constant of water from 0 to 100 C. J. Res. Natl. Bur. Stand. 1956, 56, 1.

(47) Sengwa, R. J.; Kaur, K.; Chaudhary, R. Dielectric properties of low molecular weight poly(ethylene glycol)s. Polym. Int. 2000, 49, 599-608.

(48) Arnold, K.; Herrmann, A.; Pratsch, L.; Gawrisch, K. The dielectric properties of aqueous solutions of poly(ethylene glycol) and their influence on membrane structure. Biochim. Biophys. Acta Biomembr. 1985, 815, 515-518.

(49) Karlstroem, G. A new model for upper and lower critical solution temperatures in poly(ethylene oxide) solutions. J. Phys. Chem. 1985, 89, 4962-4964.

(50) Bjoerling, M.; Karlstroem, G.; Linse, P. Conformational adaption of poly(ethylene oxide): A carbon-13 NMR study. J. Phys. Chem. 1991, 95, 6706-6709.

(51) Polik, W. F.; Burchard, W. Static light scattering from aqueous poly(ethylene oxide) solutions in the temperature range $20-90^{\circ} \mathrm{C}$. Macromolecules 1983, 16, 978-982.

(52) Ataman, M.; Boucher, E. A. Properties of aqueous salt solutions of poly(ethylene oxide). J. Polym. Sci. Polym. Phys. Ed. 1982, 20, $1585-1592$.

(53) Ananthapadmanabhan, K. P.; Goddard, E. D. Aqueous biphase formation in polyethylene oxide-inorganic salt systems. Langmuir $1987,3,25-31$.

(54) Boucher, E. A.; Hines, P. M. Effects of inorganic salts on the properties of aqueous poly(ethylene oxide) solutions. J. Polym. Sci. Polym. Phys. Ed. 1976, 14, 2241-2251.

(55) Petsev, D. N.; Denkov, N. D.; Kralchevsky, P. A. Flocculation of Deformable Emulsion Droplets. J. Colloid Interface Sci. 1995, 176, 201-213.

(56) Paula, S.; Sues, W.; Tuchtenhagen, J.; Blume, A. Thermodynamics of Micelle Formation as a Function of Temperature: A High Sensitivity Titration Calorimetry Study. J. Phys. Chem. 1995, 99, 11742-11751.

(57) Duplâtre, G.; Ferreira Marques, M. F.; da Graça Miguel, M. Size of Sodium Dodecyl Sulfate Micelles in Aqueous Solutions as Studied by Positron Annihilation Lifetime Spectroscopy. J. Phys. Chem. 1996, 100, 16608-16612.

(58) Shah, S. S.; Jamroz, N. U.; Sharif, Q. M. Micellization parameters and electrostatic interactions in micellar solution of sodium dodecyl sulfate (SDS) at different temperatures. 2001, 178, 199-206. DOI: 10.1016/s0927-7757(00)00697-x

(59) Sainath, K.; Ghosh, P. Stabilization of Silicone Oil-in-Water Emulsions by Ionic Surfactant and Electrolytes: The Role of Adsorption and Electric Charge at the Interface. Ind. Eng. Chem. Res. 2013, 52, 15808-15816.

(60) Koh, A.; Gillies, G.; Gore, J.; Saunders, B. R. Flocculation and Coalescence of Oil-in-Water Poly(dimethylsiloxane) Emulsions. J. Colloid Interface Sci. 2000, 227, 390-397.

(61) Segrè, P.; Prasad, V.; Schofield, A.; Weitz, D. Glasslike Kinetic Arrest at the Colloidal-Gelation Transition. Phys. Rev. Lett. 2001, 86, 6042-6045.

(62) Joshi, Y. M. Dynamics of Colloidal Glasses and Gels. Annu. Rev. Chem. Biomol. Eng. 2014, 5, 181-202.

(63) Cardinaux, F.; Gibaud, T.; Stradner, A.; Schurtenberger, P. Interplay between spinodal decomposition and glass formation in proteins exhibiting short-range attractions. Phys. Rev. Lett. 2007, 99, $1-4$. 